Archivum, LXIV, 2014, pp. 217-238

\title{
Hibridismo y Primera Guerra Mundial: realidad, ficción y géneros literarios en The Cellar-House of Pervyse (1916)
}

Recibido: 12/02/2014

Aceptado: 30/05/2014

\begin{abstract}
RESUMEN:
Este artículo examina el juego literario entre realidad y ficción que se desarrolla en The Cellar-House of Pervyse (1916). El libro narra la labor desarrollada por Elsie Knocker y Mairi Chisholm, "las mujeres de Peroyse", en un hospital de campaña durante los primeros meses de la Primera Guerra Mundial. Jugando con los límites entre la escritura de ficción, el género epistolar y la literatura autobiográfica, la autora-editora del texto, Geraldine Mitton, construye una inusual narrativa que permite ilustrar la dificultad a la que muchos autores/as se enfrentaron a la hora de narrar y describir una guerra devastadora que rompió con los parámetros de representación de la guerra previamente establecidos.
\end{abstract}

PALABRAS CLAVE: Géneros literarios, Primera Guerra Mundial, escritura testimonial.

\section{ABSTRACT:}

This article examines the literary game between fiction and reality that takes places in The Cellar-House of Pervyse (1916). The book describes the role that Elsie Knocker and Mairi Chisholm, "the women of Peroyse", developed in a field hospital during the 
early months of the First World War. Geraldine E. Mitton (the author-editor of the book) plays with the limits of fiction, epistolary and autobiographical writing, to construct an unusual narrative. As a result, the text illustrates the difficulties that many authors faced when describing a devastating war that broke all previously established parameters of representation.

KEY WORDS: Literary genres, First World War, testimonial literature.

Es sabido que la Primera Guerra Mundial tuvo un componente especialmente liberador para las mujeres. Aunque para muchas de ellas la guerra significó un tremendo sacrificio personal, con la pérdida de hijos, maridos, hermanos y amigos en el campo de batalla, a nivel colectivo el conflicto conllevó la emancipación de la mujer en muchos ámbitos. La guerra permitió el acceso de la mujer a puestos de trabajo antes vedados y su participación en el desarrollo de la vida en la retaguardia contribuyó a que los países beligerantes pudieran seguir produciendo mientras los hombres luchaban en las trincheras. Además, con el estallido de la Primera Guerra Mundial muchas mujeres se embarcaron en lo que el periodista británico Philip Gibbs ha llamado "the call of the wild": atraídas por la posibilidad de ver el desastre de la guerra y, de paso, contribuir a auxiliar a los combatientes, numerosas mujeres se ofrecieron voluntarias para participar como enfermeras o conductoras de ambulancia en cuerpos humanitarios como la Cruz Roja ${ }^{2}$. La presencia de mujeres en la primera línea de fuego y sus proximidades estaba oficialmente prohibida. En la práctica, sin embargo, muchas lograron trabajar en hospitales

1 "la llamada de lo salvaje". Gibbs, Philip, The Soul of the War, New York: A.L. Burt Company, 1919, p. 214.N. del T.: Todas las traducciones son de la autora del artículo.

2 El artículo de Sandra Gilbert $<<$ Literary Men, Literary Women and the Great War $>$ reflexiona sobre el componente liberador que la Guerra tuvo para muchas mujeres y las consecuencias literarias que esta liberación supuso. Gilbert, Sandra, <<Literary Men, Literary Women and the Great War $>$, Signs, 8, 3, 1983, págs. 422450 . 
o estaciones de campaña en las zonas próximas al campo de batalla ${ }^{3}$.

En el campo literario, el estallido de la Primera Guerra Mundial también supuso una revolución. El conflicto marcó un antes y un después en torno al discurso bélico en la literatura anglosajona, pues a través de los conocidos "soldados-poetas" como Wilfred Owen o Sigfried Sassoon se destruyó el mito de que la guerra era algo honroso y heroico. Estos escritores dieron voz a "the experience of the trenches" y sus textos, centrados en la experiencia del combatiente, formaron el canon literario en torno a la Gran Guerra hasta que en la década de los ochenta y noventa se empezaron a recuperar numerosos textos escritos por mujeres que también fueron testigos directos del conflicto ${ }^{4}$. El motivo por el que los textos escritos por mujeres se mantuvieron apartados de los estudios de la Primera Guerra Mundial fue, precisamente, que no giraban en torno a la figura del combatiente o al trauma de la vida en las trincheras ${ }^{5}$. Tal y cómo explica Margaret Higonnet en Lines of Fire, los textos escritos por mujeres se consideraban "'inauthentic' and unrealistic, since they stood -even if only symbolically- outside the line of fire ${ }^{6 \prime \prime}$.

Sin embargo, son numerosos los textos escritos por mujeres que presentan una lectura del conflicto que da cuenta de la realidad literaria del momento en que fueron escritos y de las di-

\footnotetext{
3 Grayzel, Susan R. $<<$ Women and Men $\gg>$, A Companion to World War I. Malden, Blackwell Publishing, 2010, p. 268.

4 "la experiencia de las trincheras". Higonnet, Margaret et al. <<Introduction〉>, Behind the Lines: Gender and the Two World Wars. Yale, Yale University, 1987, p. 13.

5 Smith, Angela K. The Second Battlefield: Women, Modernism and the First World War. Manchester, Manchester University Press, 2000, p. 3.

6 "la escritura de las mujeres se entendía como "inauténtica" e irreal, ya que se encontraban -aunque sólo fuera simbólicamente-fuera de la línea de combate. "Higonnet, Margaret. Lines of Fire: Women Writers of World War I. New York, Penguin Books, 1999, p. xxiii.
} 
ficultades de representación a las que se enfrentaron las mujeres que trataron de articular su experiencia bélica. The Cellar-House of Pervyse es uno de estos textos. Publicado en 19167, The CellarHouse of Pervyse se centra en la historia de Elsie Knocker y Mairi Gooden-Chisholm, dos enfermeras británicas que en septiembre de 1914 se enrolaron en el Motor Ambulance Corps, una unidad de emergencia independiente que un médico escocés, Hector Munro, había organizado para socorrer a los heridos en el frente occidental de Bélgica ${ }^{8}$. Geraldine Mitton fue la encargada de editar y publicar la historia de estas dos mujeres en Bélgica a partir de los diarios que ambas habían escrito por su propio interés personal, sin ningún afán de publicación ${ }^{9}$.

El texto está construido a partir de las cartas y los diarios que ambas autoras escribieron. Sin embargo, no mantiene la estructura original de los diarios, lo cual dota al texto de unas características únicas frente a muchas de las publicaciones escritas por mujeres durante el mismo periodo. Los diarios y las

7 El almacén de Pervyse: un relato de sucesos extraordinarios a través de los diarios y cartas de la Baronesa T'Serclaes y Mairi Chisholm.

8 Elsie Knocker (1884-1978), más tarde Baronessa T'Serclaes y Mairi Chisholm (1896-1981), son conocidas como "Las dos mujeres de Pervyse", "Las heroínas de Pervyse" o "Las Madonnas de Pervyse". Además de ser conductoras y enfermeras su fama les vino sobre todo por el famoso almacén que construyeron a pocos metros de la primera línea de fuego en el frente belga cerca de Yser donde socorrieron a cientos de soldados heridos. Gómez Reus, Teresa, $<<$ Fighting for Fame: The 'Heroines of Pervyse' and the Disputed Construction of a Public Image $\gg$, Women: A Cultural Review 23, 3, 2012, p. 301.

9 Tal y como nos indica Diane Atkinson, cuando a principios de 1916 Knocker y Chisholm estaban buscando fondos para subvencionar sus actividades en el frente, el comandante A.A. Gordon sugirió la publicación de un libro narrando sus experiencias en Pervyse. Los diarios fueron entregados a Geraldine Mitton, autora relativamente conocida en su tiempo, para que los editara y publicara. El libro se publicó en noviembre de ese mismo año y el dinero que obtuvieron por los derechos lo destinaron a comprar una nueva moto ambulancia y para pagar el trabajo en Pervyse. Atkinson, Diane, Elsie\&Mairi Go to War. Two Extraordinary Women on the Western Front, London, Arrow Books, 2010, págs. 158-159. 
cartas personales fueron las primeras formas de escritura que las mujeres utilizaron para narrar su experiencia en el frente. Estos, explica Gómez Reus, "permitieron a las mujeres expresarse con una espontaneidad y una autenticidad muy difíciles de encontrar en la escritura que se sabe pública ${ }^{10 \prime}$. A su vez, este género literario ha supuesto un gran reto para investigadores y críticos literarios e historiográficos pues la complejidad (y en ocasiones indefinición genérica) de las cartas y los textos autobiográficos supone un obstáculo para su clasificación y estudio. En "The Diary between Literary and History: A Historian's Critical Response", Jochen Hellbeck reflexiona:

The diary has bedeviled literary and historical scholars alike. Its "uncertain" nature between literary and historical writing, between fictional and documentary, spontaneous and reflected narrative, has frustrated many a literary specialist in search for canonical identity. At least equally frustrating has it proved for historians to work with the diary's personal testimony, which promises sincere, private testimony, yet which at closer sight reveals manifold connections to conventions governing the public world ${ }^{11}$.

En el caso de The Cellar-House esta "uncertain nature" del diario se vuelve aún más compleja, pues no estamos ante una representación literal de los diarios de las dos autoras, sino ante una

10 Gómez Reus, Teresa. ¡Zona Prohibida! Mary Borden: Una enfermera norteamericana en la Gran Guerra.Valencia, Universidad de Valencia, 2011, págs. 20-21. Esta idea está ampliamente desarrollada en Smith, A. The Second Battlefield..., págs. 46-69.

11 "El diario ha sido un problema tanto para los estudiosos de la literatura como para los historiadores. Su naturaleza "incierta", a medio camino entre lo literario y lo histórico, lo ficcional y lo documental, lo espontáneo y lo reflexivo, ha frustrado a más de un especialista literario que buscaba una identidad canónica. Al menos igual de frustrante ha sido para los historiadores el trabajar con testimonios personales de diarios, que se presentan como testimonios sinceros y privados, pero que en un análisis más profundo presentan diversas conexiones con las convenciones que gobiernan el contexto en que son escritas."Hellbeck, Jochen, $<<$ The Diary between Literature and History: A Historian's Critical Response. $>$, The Russian Review, 6.4, 2004, p. 621. 
adaptación de los mismos. Mitton no se ha limitado a transcribir los diarios de las dos mujeres de Pervyse, sino que los ha editado y unido en un único hilo argumental, creando así una especie de collage literario en el que, como veremos, el juego entre la realidad y la ficción tendrán un papel fundamental.

El título completo de la obra, The Cellar-House of Peroyse: A Tale of Uncommon Things from the Journals and Letters of the Baroness T'Serclaes and Mairi Chisholm, ya nos anticipa la ambigüedad a la que nos vamos a enfrentar a la hora de abordar el texto, pues la autora-editora, Geraldine Mitton, nos advierte de que estamos ante un "relato de cosas poco comunes" cuyo origen está en los "diarios y cartas" de las que fueron conocidas como las Mujeres de Pervyse. La inclusión de la palabra "relato", generalmente asociado al mundo de lo ficticio, combinada con la referencia a los "diarios y cartas", asociados tradicionalmente al mundo de lo real, desvela un conflicto que se acentúa en el momento en que descubrimos que las dos protagonistas de la narración son dos personajes históricos reales y no imaginarios. Mitton clasifica The Cellar-House of Peroyse como un relato, pero nos advierte de que todo lo que allí se narra ha sido extraído exclusivamente de los diarios y cartas de las dos mujeres. De este modo parece estar presentando unos datos que se antojan como reales y verídicos, como lo que se podrían considerar hechos históricos. Sin embargo, en la nota inicial que incluye al principio de la obra nos

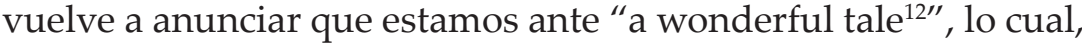
al igual que sucede con el título, nos hace dudar de la naturaleza real o fantástica del texto.

Esta cuestión se vuelve aún más compleja cuando Mitton mezcla el hecho real acontecido -la experiencia de las dos mujeres en el hospital improvisado de Pervyse- con la adaptación que realiza del mismo en su labor como editora del texto. Mitton

12 "Un relato maravilloso". Mitton, Geraldine E., The Cellar-House of Pervyse: A Tale of Uncommon Things from the Journals and Letters of the Baroness T'Serclaes and Mairi Chisholm, London, A\&C Black, 1917, p. viii. 
nos anuncia en un principio que: "The facts are so astounding that they need no dressing. My part has been merely that of a recorder, running the two parallel journals together and omitting repetitions or details too small to be of general interest $t^{13 \prime}$. No obstante, Mitton presenta una evidente contradicción en su argumento, pues reconoce que aunque haya sido por pura necesidad editorial (presuntamente estilística) ha decidido omitir algunos fragmentos que ella considera que no son de interés general para el lector. Esta anotación, sumada al hecho de que no se ha limitado a publicar los dos diarios, sino que los ha editado y unido, creando así una única línea narrativa, hace del texto un testimonio de dudosa fiabilidad histórica pero, a su vez, aporta elementos relevantes para su estudio como texto literario.

Aunque la autora niega haber realizado una adaptación de los textos, la narración fluctúa entre un "yo narrativo" presente en el primer capítulo - presuntamente la voz implícita de la propia Mitton- que nos informa de la primera vez que vio a las dos mujeres de Pervyse, Mairi Chisholm y Elsie Knocker ${ }^{14}$, y un narrador omnisciente en tercera persona, supuestamente objetivo, que narra lo que les sucedió a estas dos mujeres ${ }^{15}$. Es precisamente esta tercera persona la que dominará la narración, encontrando la perspectiva directa de las protagonistas de la historia $-\mathrm{y}$ autoras de los textos originales- sólo en ocasiones muy concretas. De hecho, la primera reproducción literal de uno de los textos escritos por Chisholm y Knocker no aparece hasta el capítulo II: una carta de la futura Baronesa de T'Serclaes donde narra su

13 “Los hechos son tan impresionantes que no necesitan ningún adorno. Mi papel ha sido exclusivamente el de cronista, uniendo los diarios paralelos y omitiendo repeticiones o detalles demasiado insignificantes como para ser de interés general. "Mitton, G., The Cellar-House..., p. x.

14 "I shall never forget them as I saw them first" ("Nunca olvidaré cómo las vi por primera vez..."). Ibid., p.1.

15 Ibid., p.8. 
primera experiencia bajo fuego ${ }^{16}$, el 5 de octubre de 1914, la cual fue publicada en un periódico local, según nos indica Mitton ${ }^{17}$. Si bien es cierto que la autora no llega a utilizar el estilo indirecto libre o el flujo de conciencia característico del movimiento modernista en lengua inglesa, sí que presenta unos cambios en la voz narrativa que no siguen los cánones de la narración realista. En este sentido el texto presenta un carácter heterógeneo que coincide con el de otros textos escritos por mujeres en el mismo periodo, los cuales, según Angela K. Smith, "can provide an interface between some of the realist literary traditions of the nineteenth century and the more experimental forms of the $1920 \mathrm{~s}^{18 \prime \prime}$.

Mitton incorpora a su vez a la narración reproducciones de diálogos en estilo directo. Al final del Capítulo V nos relata el encuentro entre las dos mujeres y un soldado francés que se presentó en el hospital de campaña una mañana en busca de su revólver:

He explained hurriedly and with his eyes anywhere but on her face that he wanted his revolver. "But I have not got your revolver" said Mairi, too surprised for words. "Gipsy, here's a man who wants his revolver - at least, I think that's what he wants, unless there is some other French word which sounds exactly like it ${ }^{19 \prime}$.

La inclusión de este estilo directo nos remite a la discusión sobre la nota inicial escrita por Mitton, pues si ésta se ha limita-

16 Ibid., p.25.

17 Ibid., p. 25-28.

18 "puede crear una interconexión entre algunas de las tradiciones del realismo literario del siglo XIX y las formas más experimentales de los años veinte". Smith, A. The Second Battlefield..., p.4.

19 “Explicó apresuradamente y con la mirada en cualquier sitio, menos en su cara, que quería su revólver. 'Pero no tengo tu revólver', dijo Mairi, demasiado sorprendida como para poder hablar. 'Gipsy, aquí hay un hombre que quiere su revólver - al menos creo que eso es lo que quiere, salvo que haya otra palabra en francés que suene exactamente igual.' "' Ibid., p.83. 
do exclusivamente a hacer el papel de cronista tenemos que preguntarnos cómo se explica la existencia de un diálogo en medio del relato histórico. Sin duda, podemos no sólo sospechar sino afirmar que no estamos ante una reproducción objetiva y fiel de los diarios y cartas de Chisholm y Knocker sino ante una ficcionalización y adaptación de los mismos.

A medida que avanza el relato descubrimos que Mitton no sólo no está limitándose a transcribir los dos diarios, sino que está construyendo el relato utilizando varias fuentes, más allá de las dos indicadas inicialmente. Mitton incorpora al relato fragmentos de cartas y reportajes de otras mujeres que fueron testigos de los acontecimientos narrados en The Cellar-House of Pervyse. Son tres, al menos, las ocasiones en que Mitton cita o reproduce lo escrito por otras autoras del mismo período. En el capítulo IX introduce fragmentos de una carta escrita por Miss Macnaughtan ${ }^{20}$; en el capítulo XII hace referencia a la escritora norteamericana Mary Roberts Rinehart ${ }^{21}$, quien se encontró con las dos mujeres y escribió sobre ellas en Kings, Queens, and Pawns: An American Woman at the Front (1915); y en el capítulo XIII reproduce literalmente una publicación de la escritora británica May Sinclair en el Daily Chronicle ${ }^{22}$. Su relato deja por tanto de tener la relevancia histórica que ella quiere otorgarle y se convierte en un collage literario donde encontramos, a modo de palimpsesto, trazas de otros textos, ya sean diarios, cartas o recortes de periódicos, que

20 Ibid. p.193. Macnaughtan, Sarah Broom , (1864-1916), enfermera y escritora. Al comienzo de la Primera Guerra Mundial se encontraba en Canadá, desde donde regresó al Reino Unido para alistarse y acudir a Bélgica con la unidad del Hospital Mabel Annie St.Clair Stobart.

21 Ibid. p. 202. Rinehart, Mary Roberts (1876-1958), periodista y autora norteamericana, enviada especial del Saturday Evening Post en Inglaterra, Bélgica y Francia a principios de 1915 .

22 Ibid. p. 210. Sinclair, Mary Amelia St Clair (1863-1946), novelista y filósofa. En el otoño de 1914 se unió durante tres semanas al cuerpo de ambulancias del Doctor Hector Munro, donde trabajó como secretaria, tesorera y publicista. De esta aventura publicaría A Journal of Impressions in Belgium (1914). 
están contribuyendo a la creación de una historia diferente a la plasmada en los diarios de Chisholm y Knocker.

La autora ha adornado los textos originales para hacerlos más accesibles al lector. Tal y como expresa Claire Tylee en su influyente estudio The Great War and Women's Consciousness: Images of Militarism and Womanhood in Women's Writings: 1914-1964, Mitton hizo "what 'splendour and romance' she could out of the brief diaries kept by the two 'Heroines of Pervyse', but they provided meagre material ${ }^{23 \prime}$. No obstante, Tylee no se detiene a analizar los recursos que Mitton empleó para adornar el escaso material que los diarios le brindaban ni tampoco estudia las contradicciones en las que cae la autora a la hora de presentar su texto ante el lector. Es aquí precisamente donde, según mi opinión, reside la riqueza de The Cellar-House of Pervise, y es en este aspecto en el que me propongo ahondar en este trabajo.

En este sentido, el primer aspecto que podemos destacar es el hecho de que en más de una ocasión la voz narrativa se ve obligada a aclarar los motivos por los que ha decidido omitir o incluir determinados fragmentos y así poder justificar su particular versión histórica de los diarios. De este modo, en el capítulo XII la autora-editora vuelve a romper el ritmo narrativo para anunciarnos que "the urgency of the work compelled Gipsy to break off her journal, which does not begin until September; but sturdy Mairi continued hers, and Gipsy's letters home fill the gap $^{24 \prime}$. Mitton interrumpe su relato, de nuevo siguiendo una técnica poco realista y más acorde con el fragmentado estilo modernista, para aclarar las decisiones editoriales que ha llevado a cabo

23 "el 'esplendor y el romance' que pudo a partir de los breves diarios de las dos 'Heroínas de Pervyse', pero estos proveyeron material escaso". Tylee, Claire. The Great War and Women's Consciousness: Images of Militarism and Womanhood in Women's Writings: 1914-1964, Iowa, University of Iowa Press, 1990, p.32.

24 "la urgencia en el trabajo obligó a Gipsy a interrumpir su diario, que no empieza hasta septiembre, pero Mairi, tenaz, siguió con el suyo; y las cartas de Gipsy a su casa, ayudan a rellenar este hueco". Ibid., p. 140. 
y, a su vez, para recordarnos que está narrando hechos históricos basándose exclusivamente en fuentes primarias. Es decir, vuelve a jugar con el lector, dando cuenta de los referentes del mundo real que ha utilizado -los diarios y cartas- para dotar a su historia de verosimilitud y para otorgarle un carácter más veraz.

En segundo lugar, directamente vinculado con las irrupciones de la voz narrativa que comentamos, tampoco nos queda claro el punto de vista desde el cual está narrada la historia, pues Mitton se adueña en más de una ocasión de las aseveraciones y opiniones vertidas por Chisholm y Knocker en sus diarios originales. En el capítulo III, por ejemplo, narra la actuación de las mujeres de Pervyse en el pequeño pueblo de Melle, a unos veinte kilómetros de Gante, a finales de octubre de 1914, donde "the Two were to have one of the greatest experiences of their lives one of those experiences which scores a deep mark across consciousness, so that the 'after' can never again be quite as the 'before' ${ }^{25}$ '. En el pueblo estaba teniendo lugar un brutal enfrentamiento entre belgas y alemanes que causó numerosas víctimas en ambos bandos. Este episodio es de especial relevancia por dos motivos. En primer lugar, por la significancia del impacto psicológico de este enfrentamiento en Chisholm y Knocker. La idea de que el después no puede ser igual que el antes en la conciencia de los dos personajes se relaciona directamente con la idea de rito de paso que Eric Leed asocia con la experiencia bélica de los combatientes: la Gran Guerra como experiencia liminal en la que el soldado sufrió un proceso trasformador y de aprendizaje ${ }^{26}$. No sólo fueron los soldados, sino todos aquellos que fueron testigos directos del horror de la guerra quienes sufrieron esta traumática transformación psicológica.

25 “Aquí las dos mujeres tuvieron una de las experiencias más grandes de su vida, de las que dejan una huella profunda en la consciencia, de modo que 'después' nunca puede ser como 'antes.'"Ibid., p.33.

26 Leed, Eric. No Man's Land: Combat E Identity in World War I. Cambridge, Cambridge University Press, 1979, 12-33. 
En segundo lugar, esta descripción está construida como si Mitton también hubiera sido testigo directo de la masacre. La editora nos indica que "the village was a village of horrors" ${ }^{27 "} . \mathrm{Si}$ bien es cierto que es más que probable que Mitton haya extraído esta afirmación de alguno de los diarios de Chisholm y Knocker, al no citarla como una reflexión directa de las dos mujeres y añadirlo a la narración como si fuera su propio punto de vista, nos hace sentir que la narradora se está adueñando de la historia, convirtiéndose en una narradora omnisciente y, en cierto modo, ficcionalizando el relato. De este modo, Mitton se sitúa en la posición del narrador omnisciente, haciendo creer al lector que ella también fue testigo de la barbarie acontecida en Melle y que ella también sufrió las consecuencias psicológicas de haber sido testigo de ese episodio. Esta idea se puede relacionar con un oculto deseo de la propia Mitton de experimentar por sí misma estos acontecimientos: como indicamos al principio, la guerra brindó la oportunidad a numerosas mujeres de acceder a un terreno vedado física y literariamente y Mitton no fue una de las mujeres "privilegiadas" en acceder al frente. Como editora se tuvo que contentar con vivir esta experiencia de modo vicario, a través de los diarios de Knocker, Chisholm y otras mujeres que sí pudieron adentrarse en la experiencia liminal ${ }^{28}$.

No podemos olvidar que el contexto en el que fue publicado el texto es determinante para la actitud que la autora-editora adopta hacia su propio relato. Con la llegada de la Primera Guerra Mundial, tal y como indicó el historiador Eric Hobsbawn, se produjo la caída de la sociedad burguesa del S.XIX, y con ella,

27 "El pueblo era un pueblo de horrores". Ibid., p.38.

28 Teresa Gómez Reus examina en $<<$ Competing Narratives of Women in the First World War >> el elemento competitivo que se percibe en la narración de la experiencia bélica de muchas mujeres, como May Sinclair, Fryn Tennyson Jesse, Mary Roberts Rinehart o las propias Chisholm y Knocker, que ambicionaban adentrarse en la zona prohibida antes que el resto. Gómez Reus, Teresa. $<<$ Competing Narratives of Women in the First World War $\gg$, Women in Transit through Literary Liminal Spaces. New York, Palgrave, 2013, págs. 107-124. 
muchas de las creencias que sostenían al mundo ${ }^{29}$. La llegada y finalización del conflicto provocó un cambio en el modo de ver el mundo que se reflejó en la representación literaria y artística. Mitton da cuenta de estos problemas e inquietudes, preguntándose en más de una ocasión qué es la realidad y reflexionando sobre la dificultad para encontrar palabras que reflejen el horror de la contienda.

El mundo en el que se desarrolla The Cellar-House es un mundo en ruinas. Cuanto más se adentra la narradora en el relato de la experiencia bélica de las mujeres de Pervyse, mayor dimensión poética adquiere, abandonando la narración inicialmente presentada como objetiva y factual. La voz narrativa apela no sólo a los hechos acontecidos sino a las sensaciones producidas por esos hechos y a la imposibilidad de representar los mismos. En el capítulo IV, la narradora interrumpe el relato para indicar que "no words can describe the horror of the scene ${ }^{30 "}$. Esta afirmación -ligada a otras descripciones sensoriales, con constantes referencias al efecto que el silencio y el insoportable olor de los heridos causaban en Chisholm y Knocker- hace que el texto adquiera una función más poética que descriptiva. Sin embargo, una vez más, desconocemos quién es la persona que no encuentra palabras para describir el horror, pero todo apunta a que no es ni Mairi ni la Baronesa, sino la propia Mitton.

No obstante, lo verdaderamente revelador de esta afirmación es que Mitton, a partir de las reflexiones de Chisholm y Knocker, hace referencia a uno de los mayores retos literarios y estéticos a los que se tuvieron que enfrentar quienes trataron de describir los horrores de la Gran Guerra: la imposibilidad de encontrar las palabras adecuadas para representar la dimensión de la catástrofe. Esta es una constante en la literatura de la Primera Guerra

29 Hobsbawm, Eric. The Age of Extremes. The Short Twentieth Century 1914-1991. London, Abacus, 2010, p.6.

30 "no hay palabras para describir el horror de la escena". Ibid., p.63. 
Mundial, no sólo en las representaciones de quienes fueron testigos directos del conflicto sino también en quienes trataron de representarlo estéticamente. Tan solo unos meses después del estallido del conflicto, el autor norteamericano Henry James reflexionaba en una entrevista publicada en el New York Times el 21 de marzo de 1915 sobre este problema: "the war has used up words; they have weakened, they have deteriorated like motor car tires; they have, like millions of other things, been more overstrained and knocked about and voided of the happy semblance during the last six months than in all the long ages before ${ }^{31 "}$.

A su vez, a partir de esa devastadora y desgarradora desolación provocada por el conflicto, muchos de los testigos oculares de la guerra comenzaron a creer que hasta lo impensable era posible, incluso la asombrosa presencia de seres fantasmagóricos acechando detrás de las puertas. Estos elementos fantasmagóricos, pertenecientes a lo que Tomás Albaladejo denomina tipo de mundo III ${ }^{32}$, también están presentes en la narración de Mitton. Un ejemplo de esto es la cita del capítulo VII en la que Mitton indica: "Some of these tales read like the stories of knights in armour attacking dragons in the old days ${ }^{33 \prime \prime}$. En este caso, no sólo vuelve a hablar del texto como "relato" sino que compara los he-

31 "La guerra ha agotado las palabras; se han debilitado, se han deteriorado como las ruedas de los automóviles; en los últimos seis meses, igual que otros tantos millones de cosas, han sido más deformadas, golpeadas y anuladas de su feliz apariencia que en todas las eras anteriores".

32 Albaladejo considera que existen tres tipos de mundo: el de tipo I, que se corresponde con el mundo verdadero, al cual corresponderían los textos periodísticos o históricos; el de tipo II, el de lo ficcional verosímil, “cuyas reglas no son las del mundo real objetivo, pero están construidas de acuerdo con éstas"; y el modelo de mundo de tipo III donde operan las reglas que no se parecen a las del mundo real objetivo, bajo cuya categoría englobaríamos los relatos de ficción fantástica. Albaladejo, Tomás. Teoría de los mundos posibles y macroestructura narrativa: Análisis de las novelas cortas de Clarín, Alicante, Universidad de Alicante, 1998, págs. 54-84.

33 "Algunos de estos relatos son como las historias de los caballeros andantes que atacaban los dragones en el pasado". Mitton, G., The Cellar-House..., p. 96. 
chos narrados en su libro con aquellas historias de los caballeros del medievo que conforman la memoria literaria de gran parte de la población europea. La referencia a los dragones plantea la posibilidad, asimismo, de enmarcar la narración en un tipo de mundo III, puesto que los dragones no forman parte ni del mundo real objetivo (tipo I) ni del mundo ficcional verosímil (tipo II).

No será esta referencia a los dragones la única perteneciente al tipo de mundo III que Mitton introducirá en The Cellar-House. En el capítulo XIII, cuando Knocker y Chisholm se encuentran en una pequeña casa en Pervyse, la autora-editora relata cómo las dos mujeres perciben la presencia de una entidad fantasmagórica. Reproduciendo la conversación exacta, Mairi Chisholm advierte a Elsie Knocker: "Gipsy, there is something dead in here; but-yet-it's-not-dead--34". A pesar de haber sido testigos de numerosas muertes durante su tiempo como enfermeras con el cuerpo de ambulancias del Dr. Munro, las dos mujeres y el chófer que las acompaña se encuentran atemorizados ante esa inquietante e incierta presencia que no saben muy bien cómo definir:

but now they knew it was something beyond death that had awed them, something which was vague and terrible, and to all three it seemed as if this "thing" was behind that rusty barred door. It was imprisoned, yet menacing, and was trying intangibly to thrust them away. ${ }^{35}$

Nos encontramos por tanto ante un ejemplo que nos permitiría clasificar esta narración como perteneciente a un modelo 226.

34 “Gipsy, hay algo muerto ahí; pero...aún así...no...está....muerto”. Ibid., p.

35 “Pero ahora sabían que era algo del más allá lo que les había asustado, algo borroso y terrible, y para los tres era como si esa "cosa" estuviera esperando tras la puerta de barrotes. Estaba encerrado, pero era amenazante, y trataba, en su forma etérea, de alejarles de allí". Ibid., p. 227. 
de mundo tipo III, que podría cumplir la teoría de máximos semánticos expuesta por Tomás Albaladejo: es irreal, pero podría ser verosímil que hubiera espíritus extraños en la casa de Pervyse $^{36}$. No obstante, y siguiendo las restricciones expuestas por el propio Albaladejo ${ }^{37}$, el hecho de que el relato nos indique que estas inquietantes presencias son fruto de la imaginación de los personajes (como consecuencia del estrés psicológico al que estaban expuestos durante la contienda) y no de una verdadera presencia en la casa de Pervyse real en 1915, hacen que podamos seguir manteniendo el relato en la tipología del mundo II, la de lo ficcional verosímil.

Wolfang Iser reflexionó sobre el proceso de ficcionalización considerándolo "la representación formal de la creatividad humana, y como no hay límite para lo que se puede escenificar, el propio proceso creativo lleva la ficcionalidad inscrita, la estructura de doble sentido ${ }^{38 \prime}$. Si atendemos al supuesto enunciado por Iser no podemos sino afirmar que el proceso por el cual se rige Mitton (seleccionando qué fragmentos incluir, indicando al lector cómo deber leer y considerar el texto y uniendo todos los fragmentos en "a wonderful tale") no es más que un proceso de adentramiento en el mundo de lo ficticio. Si consideramos, además, que en más de una ocasión Mitton anticipa elementos que serán narrados más adelante en la historia, no cabe duda del proceso de ficcionalización que está llevando a cabo la autoraeditora y a su vez de las violaciones del género realista en las que incurre. Por ejemplo, en el capítulo IX interrumpe abruptamente la narración para comentar algo que tiene que ser narrado "in its own place ${ }^{39 \prime \prime}$, construyendo así, de acuerdo con su propio interés

36 Albaladejo. Teoría... págs.61-62.

37 Ibid. 72-74.

38 Iser, Wolfang. $<<$ La ficcionalización: Dimensión antropológica de las ficciones literarias.>>, Teorías de la ficción literaria, Arco Libros, Madrid, 1997, p.58.

39 “donde corresponde" Mitton, G., The Cellar-House..., p. 127. 
creador, una tensión narrativa más característica del género novelístico modernista que del relato realista.

Al final de la historia Mitton sigue jugando y oscilando entre lo real verdadero, lo ficcional verosímil y lo fantástico. La autora-editora concluye el libro con la boda de Elsie Knocker con el Barón T'Serclaes anunciando que "with this the book might end, as 80 per cent of the reading public consider a wedding the only satisfactory conclusion ${ }^{40 \prime}$. Mitton considera una boda el mejor final para su libro, haciendo así uso de un recurso, el del final feliz, característico de los relatos fantásticos y de los cuentos de hadas tradicionales. Mitton construye una historia a partir de fuentes reales pero ha decidido qué incluir y qué dejar fuera de su relato para darle al público lector lo que espera de semejante "relato maravilloso". Es este el motivo por el cual Mitton justifica su elección de finalizar el relato tras la boda. Si simplemente hubiera querido reproducir los eventos tal y como ocurrieron (tal y como se supone que iba a hacer, según lo anunciado al inicio del libro), podría haber omitido el episodio de la boda o haber reproducido todos los diarios literalmente, sin seleccionar y contar exclusivamente los acontecimientos más "extraordinarios" que les sucedieron a Mairi Chisholm y Elsie Knocker. En la vida real, la que se supone que está retratando Mitton, los héroes también tienen momentos de flaqueza, pero dichos momentos apenas se ven reflejados en The Cellar-House of Peroyse.

Durante el tiempo en que se desarrolla The Cellar-House of Pervyse, los referentes del mundo en paz fueron sido sustituidos por un mundo que se vio invadido por el dolor de una devastadora guerra que nada tenía que ver con las guerras libradas hasta la fecha. Pervyse -ya sea la real o la ficticia- no es el pueblo que era antaño sino que ahora está conformado por "dusty

40 "Con esto el relato llega a su fin, ya que el 80 por ciento del público lector considerará que una boda es la única conclusión satisfactoria.". Mitton, The CellarHouse..., p. 260. 
heaps and great fallen beams ${ }^{41{ }^{\prime \prime}}$. El mundo se ha roto, está descompuesto y "nothing seemed too weird for belief $f^{42 "}$. Es posible que la narradora -quien nos anuncia que conoció a Mairi y a la Baronesa- también se haya visto destruida y, en cierto modo, fragmentada, como su relato. De este modo, y tal y como hemos visto a lo largo de este artículo, Mitton hace uso -voluntaria o involuntariamente- de nuevos modos de representación para dar forma a su narración y se aleja gradualmente de la perspectiva histórica que en un principio quería otorgarle. Así, combinando elementos de los diarios y las cartas de dos mujeres que fueron testigos oculares del conflicto, apelando a referencias del imaginario colectivo y estructurando su narración como un cuento tradicional con un final feliz, Mitton construye una narración que no sólo juega con los límites entre la realidad y la ficción sino que da cuenta de los problemas a los que numerosas mujeres se enfrentaron para poder articular su experiencia bélica, ya fuera ésta vicaria o real. Esto nos remite de nuevo a la reflexión de Mitton y Henry James sobre la imposibilidad de describir con palabras el horror causado por la guerra. Es quizás ante esta ausencia de palabras donde Mitton necesita -quizá inconscientemente- recurrir a un proceso creativo que le ayude a articular lo que se dice (y lo que no se dice) en los diarios: un mundo donde se mezcle lo real y lo irreal, lo veraz y lo ficticio; un espacio de tránsito a caballo entre dos corrientes literarias en el que poder expresarse tomando fragmentos de algunos referentes reales -tales como los diarios y las cartas- pero también alejándose lo suficiente de esta realidad -a través de la ficción y de los modelos de mundo II y III- para poder encontrar la manera de representar y transmitir lo vivido por las dos mujeres de Pervyse, Elsie Knocker y Mairi Chisholm, entre 1914 y 1916.

\footnotetext{
41 "vigas de polvo y grandes vigas destruidas". Mitton, G. The Cellar-House..., p. 150.

42 "nada parecía demasiado difícil de creer". Ibid., p. 90.
} 


\section{Bibliografía}

Albaladejo Mayordomo, Tomás. Teoría de los mundos posibles y macroestructura narrativa: Análisis de las novelas cortas de Clarín, Alicante, Universidad de Alicante, 1998.

Atrinson, Diane. Elsie EMairi Go to War. Two Extraordinary Women on the Western Front, London, Arrow Books, 2010.

Blodgett, Harriet: <<Macnaughtan, Sarah Broom(18641916). >, Oxford Dictionary of National Biography. Ed. H. C. G. Matthew and Brian Harrison. Oxford, Oxford University Press, 2004. Online ed. Ed. Lawrence Goldman, 2006.14 Mayo 2013 $<$ http://www.oxforddnb.com/view/article/59332>.

Condell, Diana and Jean Liddiard. $<$ T'Serclaes, Baroness Elizabeth Blackall de (1884 1978) >>, Oxford Dictionary of National Biography. Ed. H. C. G. Matthew and Brian Harrison. Oxford, Oxford University Press, 2004. Online ed. Ed. Lawrence Goldman, 2006.20 abril2013<http://www.oxforddnb.com/view/ article/67675>.

$:<<$ Chisholm, Mairi Lambert Gooden, of Chisholm (1896-1981) >>, Oxford Dictionary of National Biography. Ed. H. C. G. Matthew and Brian Harrison. Oxford, Oxford University Press, 2004. Online ed. Ed. Lawrence Goldman, 2006.20 abril 2013<http://www.oxforddnb.com/view/ article/67674>.

Gómez Reus, Teresa. <<Competing Narratives of Women in the First World War $\gg$, Women in Transit through Literary Liminal Spaces. New York, Palgrave, 2013, págs. 107-124.

Gómez Reus, Teresa. <<Fighting for Fame: The 'Heroines of Pervyse' and the Disputed Construction of a Public Image $\gg$, Women: A Cultural Review 23, 3, 2012, págs. 300-322.

Gómez Reus, Teresa. ¡Zona Prohibida! Mary Borden: Una enfermera norteamericana en la Gran Guerra.Valencia, Universidad de Valencia, 2011. 
Gibbs, Philip. The Soul of the War, New York: A.L. Burt Company, 1919.

Gilbert, Sandra. <<Literary Men, Literary Women and the Great War $>$, Signs, 8, 3, 1983, págs.422-450.

Grayzel, Susan R. $<<$ Women and Men>>, A Companion to World War I, John Horne, ed., Malden, Blackwell Publishing, 2010, págs. 263-278.

Hobsbawm, Eric. The Age of Extremes. The Short Twentieth Century 1914-1991. London, Abacus, 2010.

Hellbeck, Jochen. <<The Diary between Literature and History: A Historian's Critical Response $\gg$, The Russian Review, 63.4, 2004, págs. 621-629.

Higonnet, Margaret. Lines of Fire: Women Writers of World War I. New York, Penguin Books, 1999.

Higonnet, Margaret, Jane Jenson, Sonya Michel, Margaret Collins. <<Introduction>>, Behind the Lines: Gender and the Two World Wars. Yale, Yale University, 1987, págs. 1-17.

Iser, Wolfang. <<La ficcionalización: Dimensión antropológica de las ficciones literarias $\gg>$, Teorías de la ficción literaria 43-69. Antonio Garrido, ed., Madrid, Arco Libros, 1997, págs. 43-69.

Leed, Eric. No Man's Land: CombatEIdentity in World War I. Cambridge, Cambridge UniversityPress, 1979.

Mitton, Geraldine E. The Cellar-House of Peroyse: A Tale of Uncommon Things from the Journals and Letters of the Baroness T'Serclaes and Mairi Chisholm, London, A\&C Black, 1917.

Rinehart, Mary Roberts. Kings, Queens and Pawns: An American Woman at the Front. New York, George Doran Company, 1915.

Saunders, Max. <<Sinclair, Mary Amelia St Clair (18631946)>>. Oxford Dictionary of National Biography. Ed. H. C. G. Matthew and Brian Harrison. Oxford, Oxford University Press, 
2004. Online ed. Ed. Lawrence Goldman, 2006.14 Mayo 2013 <http://www.oxforddnb.com/view/article/37966>.

Sinclair, May. A Journal of Impressions in Belgium. London, MacMillan Company, 1915.

Smith, Angela K. The Second Battlefield: Women, Modernism and the First World War. Manchester, Manchester University Press, 2000.

Tylee, Claire. The Great War and Women's Consciousness: Images of Militarism and Womanhood in Women's Writings: 1914-1964, Iowa, University of Iowa Press, 1990.

Sara Prieto García-Cañedo

Universidad de Alicante 
